\title{
THE ASYMPTOTIC LINES OF THE KUMMER SURFACE.
}

\author{
BY DR. J. I. HUTCHINSON.
}

(Read before the American Mathematical Society at the Meeting of April 29, 1899.)

THE asymptotic lines of the Kummer surface were first determined by $\mathrm{Lie}^{*}$ by means of a transformation which establishes a correspondence between two spaces such that to the points of one space correspond the lines of a complex in the other. These curves have been the object of several other investigations, $\uparrow$ all of which have treated the problem from the point of view of line geometry.

A very simple solution of the problem can be obtained without the aid of the line geometry by making use of the parametric representation of the Kummer surface in terms of hyperelliptic functions. Among the indefinite number of such representations that are possible, the one most convenient for the purpose is that given by Weber, by which the homogeneous coördinates of a point on the surface are taken proportional to four linearly independent theta functions of two variables $u_{1}, u_{2}$, of the second order and characteristic zero. The equation of a tangent plane can then be written

$$
\vartheta(u+v) \vartheta(u-v)=0
$$

where $\vartheta(w)\left(=\vartheta\left(w_{1}, w_{2}\right)\right)$ is a function of the first order and any characteristic, and $v_{1}, v_{2}$ are arbitrary constants. These latter may in fact be regarded as the tangential parameters for the Kummer surface in a sense the exact dual of that in which $u_{1}, u_{2}$ are the point parameters.

The two factors in (1) separately equated to zero represent the same curve, although discriminating between the two branches which pass through the point of contact of the

*S. Lie, "Sur une transformation géométrique," Comptes rendus, vol. $71(1870)$, p. 579.

†Klein und Lie, "Ueber die Haupttangentencurven der Kummer'schen Fläche," Monatsber. der Berl. Akad., 1870, p. 891 ; Reye, "Ueber die Singularitätenflächen, etc.," Crelle, vol. 97, p. 284 ; Segre, "Sur les courbes de tangents principales, etc.," Crelle, vol. 98, p. 301; Rohn, "Transformation der hyperelliptischen Functionen, etc.," Math. Annalen, vol. 15, p. 315 .

‡Cf. Humbert, "Théorie générale, etc.," Liouville's Journal, 1893, p. 112 . 
tangent plane. The parameters for this point are the values of $u_{1}, u_{2}$ which satisfy the two equations

$$
\begin{aligned}
& \vartheta(u+v)=0, \\
& \vartheta(u-v)=0,
\end{aligned}
$$

simultaneously.

Let $u_{1}+d u_{1}, u_{2}+d u_{2}$ be the coördinates of a consecutive point on that branch of the curve represented by the equation $\vartheta(u+v)=0$. We have then

$$
\vartheta(u+d u+v)=0,
$$

or, expanding in powers of $d u_{1}, d u_{2}$ and dropping infinitesimals of higher order,

$$
\vartheta_{1}(u+v) d u_{1}+\vartheta_{2}(u+v) d u_{2}=0
$$

where

$$
\vartheta_{i}(u+v)=\frac{\partial \vartheta(u+v)}{\partial\left(u_{i}+v_{i}\right)} .
$$

The differential equation (3) gives one of the asymptotic directions through the point of tangency $u_{1}, u_{2}$.

Suppose $v_{1}+d v_{1}, v_{2}+d v_{2}$ are the coördinates of the tangent plane at the point $u_{1}+d u_{1}, u_{2}+d u_{2}$. Then for the new point we have a pair of simultaneous equations similar to (2), viz.:

$$
\begin{aligned}
& \vartheta(u+d u+v+d v)=0, \\
& \vartheta(u+d u-v-d v)=0 .
\end{aligned}
$$

From these we derive by expansion the two following :

$$
\begin{aligned}
& \vartheta_{1}(u+v) \cdot\left(d u_{1}+d v_{1}\right)+\vartheta_{2}(u+v) \cdot\left(d u_{2}+d v_{2}\right)=0 \\
& \vartheta_{1}(u-v) \cdot\left(d u_{1}-d v_{1}\right)+\vartheta_{2}(u-v) \cdot\left(d u_{2}-d v_{2}\right)=0 .
\end{aligned}
$$

Combining the first of these with equation (3) we have

Making this simultaneous with the second equation (4) we have either

$$
\left|\begin{array}{ll}
\vartheta_{1}(u+v), & \vartheta_{2}(u+v) \\
\vartheta_{1}(u-v), & \vartheta_{2}(u-v)
\end{array}\right|=0 .
$$

or

$$
d u_{i}-d v_{i}=0 . \quad(i=1,2 .)
$$


Equation (6) determines those points whose asymptotic directions are coincident. It is, therefore, the equation of the parabolic curve. It is evident that if $v_{1}, v_{2}$ be a set of half periods, equation (6) is satisfied for all values of $u_{1}, u_{2}$ which satisfy (2). These determine the 16 conics of the Kummer surface, and as the combined degrees give exactly the right number 32 for the degree of the parabolic curve,* we have thus the complete solution of $(6)$.

For the remaining points of the Kummer surface we have by integrating ( 7 ),

$$
v_{i}=u_{i}+k_{i}, \quad(i=1,2 .)
$$

where $k_{1}, k_{2}$ are constants of integration. Substituting in (2) we have finally

$$
\begin{gathered}
\vartheta(2 u+k)=0, \\
\vartheta(k)=0 .
\end{gathered}
$$

Equation (7) †, which should more properly be replaced by

$$
\vartheta(2 u+k) \vartheta(2 u-k)=0,
$$

represents the $\infty^{1}$ asymptotic curves of the Kummer surface, the two constants $k_{i}$ being connected by the relation (8). Equation (10) shows us at a glance that each curve of the family (evidently of degree 16) has a cusp at each node of the Kummer surface, and a double point at the place corresponding to the values of $u_{1}, u_{2}$ which annul simultaneously the two factors in the left member of (10).

Cornell UNIVERsity, March, 1899.

* See Klein und Lie, loc. cit., p. 894.

†Cf. Reichardt, "Ueber die Darstellung der Kummer'schen Fläche, etc.," p. 479. 